\title{
Impact of hemodynamics and fluid energetics on liver fibrosis after Fontan operation
}

\author{
Phillip M. Trusty, MS, ${ }^{\mathrm{a}}$ Zhenglun Wei, PhD, ${ }^{\mathrm{a}}$ Jack Rychik, MD, ${ }^{\mathrm{b}}$ Pierre A. Russo, MD, ${ }^{\mathrm{c}}$ Lea F. Surrey, MD, \\ David J. Goldberg, MD, ${ }^{b}$ Mark A. Fogel, MD, ${ }^{b}$ and Ajit P. Yoganathan, $\mathrm{PhD}^{\mathrm{a}}$
}

\section{ABSTRACT}

Objective: The staged Fontan procedure has shown promising short-term outcomes in patients with single ventricles. However, Fontan-associated liver disease is a marked problem as patients age. The purpose of this study is to investigate the relationship between hemodynamics and liver fibrosis in patients undergoing the Fontan.

Methods: A total of 33 patients undergoing the Fontan with liver fibrosis were included in this study. Cardiac magnetic resonance and phase-contrast cardiac magnetic resonance data, as well as catheterization measurements and liver biopsies, were obtained for each patient. Computational fluid dynamic simulations were performed to quantify total cavopulmonary connection hemodynamics using patient-specific anatomies and blood flow waveforms reconstructed from cardiac magnetic resonance data. Collagen deposition (as a measure of liver fibrosis) was quantified by digital image analysis of Sirius Red stained slides. Statistical analyses were performed to investigate potential relationships between liver fibrosis and Fontan hemodynamics.

Results: Liver fibrosis was found to be related to global metrics (inferior vena cava flow, ventricle power output) rather than to local total cavopulmonary connection hemodynamics and efficiency. Indexed inferior vena cava flow showed a significant, positive correlation with liver fibrosis (rho $=0.624$, $P<.001)$. Upper and lower Sirius Red tertile comparisons showed a significant difference in indexed inferior vena cava flow $(P=.008)$.

Conclusions: Significant increases in inferior vena cava flow consistent with fibrosis induced arterialization and ventricular power output suggest a burden being placed on the single ventricle from liver fibrosis. Local total cavopulmonary connection flow dynamics do not seem to influence the degree of fibrosis. Favorable total cavopulmonary connection hemodynamics may not be enough to overcome the power shortage and elevated venous pressures inherent to a Fontan circulation. (J Thorac Cardiovasc Surg 2018;156:267-75)

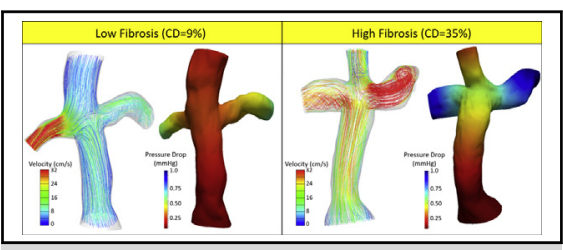

Velocity and pressure fields for low and high FALD.

Central Message

Liver fibrosis in patients with single ventricles after the Fontan is related to increased IVC flow and ventricular power output rather than local TCPC hemodynamics.

\section{Perspective}

The degree of liver fibrosis is independent of TCPC hemodynamics in patients with single ventricles with Fontan circulation. Increased IVC flow is associated with increased liver fibrosis, possibly related to hepatic arterialization, and may add a chronic volume load to the single ventricle. Superior TCPC performance may not be enough to overcome the power shortage and elevated pressures inherent to a Fontan.

See Editorial Commentary page 276.

\footnotetext{
From the ${ }^{a}$ Wallace H. Coulter Department of Biomedical Engineering, Georgia Institute of Technology and Emory University, Atlanta, Ga; ${ }^{\mathrm{b}}$ Division of Cardiology, and 'Department of Pathology and Laboratory Medicine, Children's Hospital of Philadelphia, Philadelphia, Pa.

This study and J.R.'s contributions are supported by the Robert and Dolores Harrington Endowed Chair in Pediatric Cardiology at The Children's Hospital of Philadelphia. This work was also partially supported by American Heart Association Predoctoral Fellowship 17PRE33630117.

Institutional Review Board Number 12-009791, approved on September 21, 2016.

Received for publication Sept 12, 2017; revisions received Nov 29, 2017; accepted for publication Feb 4, 2018; available ahead of print March 30, 2018.

Address for reprints: Ajit P. Yoganathan, PhD, 387 Technology Cir NW Suite 200, Atlanta, GA 30313 (E-mail: ajit.yoganathan@bme.gatech.edu). $0022-5223 / \$ 36.00$

Copyright (c) 2018 by The American Association for Thoracic Surgery https://doi.org/10.1016/j.jtcvs.2018.02.078
}

The current surgical treatment strategy for single ventricle reconstruction leads to a Fontan palliation with passive blood flow to the lungs through a total cavopulmonary connection (TCPC). Inherent physiologic differences exist between a biventricular circulatory system and a Fontan circulation, including elevated central venous pressure (CVP) and relatively diminished cardiac output $(\mathrm{CO})$, as well as a

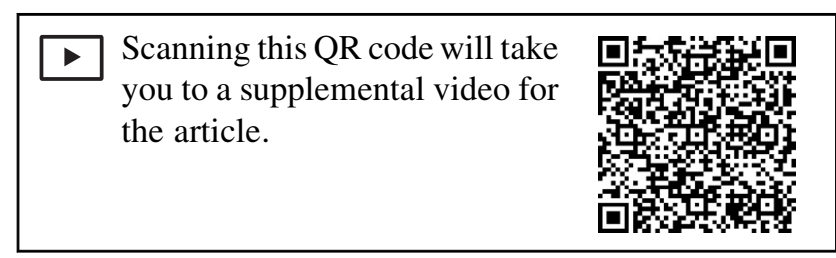




$$
\begin{aligned}
& \text { Abbreviations and Acronyms } \\
& \text { BSA }=\text { body surface area } \\
& \text { CFD }=\text { computational fluid dynamics } \\
& \text { CMR }=\text { cardiac magnetic resonance } \\
& \text { CO }=\text { cardiac output } \\
& \text { CVP }=\text { central venous pressure } \\
& \text { EDV }=\text { end-diastolic volume } \\
& \text { FALD }=\text { Fontan-associated liver disease } \\
& \text { IVC }=\text { inferior vena cava } \\
& \text { LPA }=\text { left pulmonary artery } \\
& \text { PL }=\text { power loss } \\
& \text { TCPC }=\text { total cavopulmonary connection }
\end{aligned}
$$

myriad of additional features, including the potential for ventricular dysfunction, increased vascular impedance, and venous congestion. ${ }^{1-4}$ As these unique patients survive into early adulthood, numerous long-term complications are commonly recognized, such as poor growth and development, lymphatic insufficiency manifest as protein losing enteropathy, plastic bronchitis, and liver fibrosis. $^{3,5-8}$

Fontan-associated liver disease (FALD) is a welldocumented complication that may have its roots in genetic, biochemical, and hemodynamic origins. Liver insult also may occur at birth (hypoxia and hypoperfusion), during the various perioperative states (Norwood, bidirectional Glenn and Fontan surgeries), and throughout the chronic condition of elevated CVP and relatively diminished CO. ${ }^{5}$ Regardless of cause, FALD is now recognized as "universal" and likely a progressive process affecting all Fontan survivors. ${ }^{6}$

With the goal of determining specific causes and risk factors of FALD, previous studies have investigated relationships among biochemical markers, comorbidities, cardiac morphology, demographics, hemodynamics, and hepatic fibrosis using a variety of liver assessment techniques and scores. ${ }^{6,8-11}$ Several biochemical markers show significant correlations in specific studies, as do duration of existence with a Fontan circulation and some hemodynamic measures such as hepatic wedge pressure. ${ }^{8,10-12}$ These correlations are moderate at best and often vary between studies, likely because of variability in patient cohort, sample size, and fibrosis quantification methodology.

An area left mostly untouched by these previous studies is the use of detailed cardiac magnetic resonance (CMR)derived flow dynamics and computational fluid dynamics (CFD) to assess and correlate local Fontan flows and energetics with liver fibrosis. Individual vessel flow rates could provide new information regarding factors not seen in previous studies using more global flow metrics. Investigating the energetics of the Fontan pathway and the TCPC may offer insights into surgical optimization and novel design as a method to potentially combat liver fibrosis.
To contribute to the understanding of FALD and its causes, we combine CMR, CFD, cardiac catheterization, and liver biopsy data to investigate potential relationships between hepatic fibrosis and Fontan hemodynamics. In this study, we investigated detailed CMR-derived flow dynamics and TCPC energetics as potential risk factors for hepatic fibrosis. We hypothesize that the extent of hepatic fibrosis is associated with poor Fontan hemodynamics.

\section{MATERIAL AND METHODS Patient Cohort}

A total of 33 patients with single ventricles after Fontan completion were studied with concurrent CMR, cardiac catheterization, and liver biopsy. All subjects were evaluated as part of an institutional recommended clinical care protocol for elective comprehensive assessment who are more than 10 years from their Fontan operation. ${ }^{5}$ Indication for assessment was institutional recommendation for comprehensive surveillance and not for any specific signs or symptoms of Fontan circulatory failure. No subject had any clinical signs or symptoms of hepatic dysfunction. This study cohort of 33 is a subset of a larger cohort of 67 patients who had catheterization and liver biopsy performed. Inclusion in the current cohort required that the cardiac catheterization, magnetic resonance imaging, and liver biopsy were all performed within a 6-month period. In most cases, CMR was obtained just before catheterization, and liver biopsy was performed immediately after catheterization. Demographic data including age, gender, body surface area (BSA), Fontan type, and Fontan duration were obtained for each patient. Fontan duration is defined as the time between the Fontan surgery and data collection. All patient data were obtained from the Children's Hospital of Philadelphia and collected under Institutional Review Board approval (12-009791) with written informed consent.

\section{Magnetic Resonance Imaging Acquisition}

All CMR scans were performed with a Siemens $1.5 \mathrm{~T}$ magnetic resonance imaging system (Siemens Medical Solutions, Malvern, Pa). Patients were scanned supine, head first in the scanner with electrocardiogram leads placed. After localizers were obtained, a stack of contiguous, static, diastolic steadystate free precession images were obtained from the diaphragm to thoracic inlet to assess anatomy and provide inputs for CFD modeling. Slice thickness was generally 4 to $5 \mathrm{~mm}$, and in-plane resolution was $1.2 \times 1.2 \mathrm{~mm}$.

Through-plane, retrospectively gated, phase-contrast magnetic resonance was used to assess flows in the cavae, branch pulmonary arteries, and pulmonary veins, and across the aortic valve. Inferior vena cava (IVC) flow was measured supra-hepatic. Velocity encoding was generally $150 \mathrm{~cm} / \mathrm{sec}$ for the aorta and $60 \mathrm{~cm} / \mathrm{sec}$ for the other vessels. Slice thickness was generally 4 to $5 \mathrm{~mm}$ with in-plane resolution of $1.25 \times 1.25 \mathrm{~mm}$. The number of phases was a function of the heart rate similar to the cines and ranged from 20 to 30 .

Global ventricular function was calculated from cine images of the ventricular short axis. Endocardial and epicardial borders were semiautomatically traced, and ventricular volumes were determined by multiplying the slice thickness with the segmented area. Slice volume was integrated along the ventricular longitudinal axis using Simpson's rule to extract the total volume of the ventricle in a cardiac phase. This method was applied at end diastole and end systole to calculate end-diastolic volume (EDV) and end-systolic volume, respectively. Stroke volume and ejection fraction were calculated as the EDV/end-systolic volume and stroke volume/EDV, respectively.

\section{Catheterization Protocol}

All subjects were in a fasting state for 8 hours before the catheterization, as per institutional policy. The use of light sedation, deep sedation, or 
general anesthesia was at the discretion of the catheterization team. Original pressure tracings and data from cardiac catheterization were reviewed, and measurements were repeated to ensure consistency of data interpretation for all study subjects. Protocol included obtaining a hemoglobin level 6 to 12 hours after liver biopsy with comparison with prebiopsy level and overnight observation in our postprocedure recovery unit.

\section{Hepatic Fibrosis Quantification}

Liver biopsies were obtained using ultrasound-guided percutaneous needle biopsy. Percutaneous biopsy was chosen over a transvenous approach to obtain a more representative sample of liver tissue and eliminate sampling bias in which more severe fibrosis may, theoretically, be found surrounding the hepatic veins. Anticoagulation was reversed with protamine at completion of the cardiac catheterization, and liver biopsy was performed by an interventional radiologist obtaining 1 or 2 sample cores.

Quantitative determination of hepatic fibrosis was performed by an experienced pathologist using Sirius Red staining for collagen with automated calculation of percent positive staining per slide. ${ }^{6,10}$ Slides were stained with Sirius Red and digitally scanned using Aperio Scanscope CS-O (Leica Biosystems, Vista, Calif). Whole slide image analysis with the Color Deconvolution V9 algorithm was used to calculate quantitative percent collagen deposition by automated detection of the percentage of area containing Sirius Red staining (Leica Biosystems). For subjects with biopsy specimens on multiple slides, the percentage of Sirius Red staining was averaged across all slides.

\section{Anatomic Reconstruction and Blood Flow Segmentation}

Patient-specific anatomies were reconstructed from axial CMR images using methods previously developed. ${ }^{13,14}$ Geomagic Studio (Geomagic Inc, Research Triangle Park, NC) was used to fit a surface around the reconstructed point-cloud and export the surface for mesh generation. Patient-specific blood flow waveforms were segmented from phasecontrast magnetic resonance images for all vessels of interest using previously validated methods. ${ }^{15,16}$

\section{Computational Fluid Dynamics}

The 3-dimensional segmented anatomy was imported into an ANSYS workbench, where vessel extensions of length $10 *$ (vessel diameter) were added to overcome entrance effects and establish an appropriate velocity profile. A polyhedral mesh of approximately $\mathrm{D}_{\mathrm{IVC}} / 20 \mathrm{~mm}$ elements was used to achieve mesh independent results, where $\mathrm{D}_{\mathrm{IVC}}$ is the diameter of the IVC. All simulations were performed using ANSYS Fluent (Release 17.1), which is a finite volume pressure-based Navier-Stokes solver. Blood was modeled as a single-phase Newtonian fluid $(\mu=0.04 \mathrm{~g} /(\mathrm{cm} \cdot \mathrm{s})$, $\rho=1.06 \mathrm{~g} / \mathrm{cm}^{3}$ ). Patient-specific blood flow waveforms extracted from phase-contrast magnetic resonance imaging were used as boundary conditions for each TCPC inlet and outlet. Ten cardiac cycles were simulated to overcome transition effects and achieve period stability, using the final cycle for data analysis.

\section{Data Analysis}

Calculations were performed to compute several TCPC efficiency related metrics, including power loss (PL), indexed PL, TCPC resistance, and total ventricular power output. PL was calculated from a simplified control volume approach as follows:

$$
P L=\sum_{\text {inlets }} \int_{A}\left(p+\frac{1}{2} \rho v^{2}\right) v \cdot d A-\sum_{\text {outlets }} \int_{A}\left(p+\frac{1}{2} \rho v^{2}\right) v \cdot d A
$$

where $\mathrm{p}$ is the static pressure, $\rho$ is the blood density, A is the area of the inlet/outlet, and $\mathrm{v}$ is the velocity. PL was calculated at every millisecond throughout the cardiac cycle and then averaged to obtain an overall PL value. PL was indexed as $\left(\frac{P L}{\rho Q_{s}^{3} / B S A^{2}}\right)$, and a TCPC resistance was calculated $\left(\frac{\triangle P_{T C P C}}{Q_{s}}\right)$ where $\mathrm{Q}_{\mathrm{s}}$ is the systemic venous flow $\left(\mathrm{L} \cdot \mathrm{s}^{-1}\right)$, BSA is body surface area $\left(\mathrm{m}^{2}\right), \Delta P_{T C P C}$ is the pressure drop across the TCPC, and $\rho$ is blood density $\left(\mathrm{kg} \cdot \mathrm{m}^{-3}\right) .{ }^{17}$ Total ventricular power output was defined as the flow energy produced by the single ventricle in 1 cardiac cycle. This metric was calculated as Power $_{s v}=C O * P_{a v g, s v}$ where the subscript "sv" signifies single ventricle, $\mathrm{CO}$ is cardiac output, and $P_{\text {avg }, s v}$ is the average pressure of the single ventricle. Ventricular power was converted to units of milliwatts.

The percentage of total power output lost in the TCPC was calculated as $X_{T C P C}=100 * \frac{P L}{P_{s v}}$. Pulsatility index was defined as $P I=\frac{Q_{\max }-Q_{\min }}{2 * Q_{\text {avg }}}$ where $\mathrm{Q}_{\max }, \mathrm{Q}_{\min }$, and $\mathrm{Q}_{\text {avg }}$ are the maximum, minimum, and average flow rates during the cardiac cycle, respectively, as determined from phase-contrast CMR data. Percent stenosis was calculated for each TCPC vessel as Stenosis $\%=100 *\left(\frac{A_{\text {min }}}{A_{\text {avg }}}\right)$ where $A_{\min }$ and $A_{\text {avg }}$ are the minimum and average cross-sectional areas of the vessel. All vessel areas and diameters were calculated using Vascular Modeling Toolkit version 1.0.1 (Orobix, Bergamo, Italy). Vessel diameters are calculated as the average diameter across the length of the vessel. Particle residence time was determined by seeding particles at every time step throughout 1 cardiac cycle at the IVC inlet and recording the amount of time required for $95 \%$ of the particles to exit the TCPC domain. Systemic to pulmonary collateral flow was calculated as the difference between aortic and vena cava flows, as well as the difference between pulmonary vein and pulmonary artery flow. The average of these 2 collateral flow calculations was used. Vessel diameters, flow rates, and ventricular volumes were indexed by BSA.

\section{Statistical Analysis}

SPSS (IBM Corp, Version 23, Armonk, NY) was used for statistical analyses. The Shapiro-Wilk test was used to determine normality for each parameter. Pearson's and Spearman's correlations were used to investigate bivariate correlations between liver fibrosis and hemodynamic metrics for parametric and nonparametric data, respectively. For further analysis, the data were binned on the basis of liver fibrosis score. Depending on normality, a Wilcoxon rank-sum test or a 2-sample $t$ test was used to test for equal medians between the upper and lower fibrosis score groups. A $\log$ transformation was performed to normalize variables when appropriate. A more stringent significance level of $\alpha=0.01$ was chosen to account for multiple testing. DFBETA was used to determine overly influential data points for removal. A power analysis $\left(\mathrm{G}^{*}\right.$ Power, version 3.1.9.2) revealed that an effect size of $d=1.03$ and $r=0.48$ were needed to achieve significance for the independent group and bivariate correlation tests, respectively.

\section{RESULTS}

\section{Demographic Data}

Patient demographic data are given in Table 1 . The cohort was balanced in terms of gender and Fontan type. Single ventricle morphology included $45 \%$ hypoplastic left heart syndrome and 55\% non-hypoplastic left heart syndrome. Average patient age was $16.2 \pm 4.7$ (7-26) years, with an average Fontan duration of $14.2 \pm 4.6$ (5-24) years. The average percent collagen deposition was $21.3 \% \pm 8.7 \%$ (9-49), incorporating a wide range of hepatic fibrosis. No significant correlations were found between percent collagen deposition and age, BSA, or Fontan duration. Percent collagen deposition was not significantly different 
TABLE 1. Patient demographic data $(\mathbf{n}=33)$

\begin{tabular}{lccc}
\hline & Mean \pm SD [range] & Correlation coefficient (rho) & $\boldsymbol{P}$ value \\
\hline Age $(\mathrm{y})$ & $16.2 \pm 4.7[7-26]$ & -0.055 & .766 \\
BSA $\left(\mathrm{m}^{2}\right)$ & $1.5 \pm 0.3[0.76-2]$ & 0.044 & .811 \\
Gender (male/female) & $16 / 17$ & - & .734 \\
Fontan duration (y) & $14.2 \pm 4.6[5-24]$ & -0.073 & .693 \\
Fontan type (extracardiac/intra-atrial) & $17 / 16$ & - & .236 \\
Single ventricle morphology (HLHS/non-HLHS) & $15 / 18$ & - & .457 \\
Presence of fenestration (yes/no) & $13 / 20$ & - & .803 \\
Time between CMR and liver biopsy (d) & $14 \pm 27[0-102]$ & - \\
\hline
\end{tabular}

Correlations with percent collagen deposition are shown. Categoric variables show $P$ values for the appropriate independent samples test. Values are mean \pm standard deviation [range]. Fontan duration: time since Fontan procedure. SD, Standard deviation; BSA, body surface area; HLHS, hypoplastic left heart syndrome; $C M R$, cardiac magnetic resonance.

when analyzed by gender, Fontan type, or presence of fenestration (Table 1). The average time between CMR and liver biopsy was $14 \pm 27$ days, with a range of 0 to 102 and median of 1 day.

\section{Fibrosis and Hemodynamics}

A significant, positive correlation was found between percent collagen deposition and indexed IVC flow rate (rho $=0.624, P<.001)$ (Figure $1, A)$. Likewise, indexed IVC flow rate was significantly higher $(P=.008)$ in the upper tertile of percent collagen deposition scores compared with the lowest tertile (Figure 1, B). CO was positively correlated with percent collagen deposition although not statistically significant (rho $=0.297, P=.062$ ) (Table 2). No significant correlations were found between percent collagen deposition and caval or branch pulmonary artery pressures or flow rates. In addition, there were no correlations with pulmonary venous flow rates $(\mathrm{Qp})$ or systemic to pulmonary collateral flow. Likewise, systemic vascular resistance, pulmonary vascular resistance, and IVC pulsatility index showed no significant correlations with percent collagen deposition (Table 2).

\section{Fibrosis and Ventricular Function}

Ventricular power output was positively correlated with percent collagen deposition $(\mathrm{r}=0.51, P<.01)$ (Figure 1, $C)$. Ventricular power output averaged $675 \pm 227$ (2531317) $\mathrm{mW}$. No significant correlations were seen between percent collagen deposition and EDV, end-systolic volume, stroke volume, or ejection fraction (Table 3).
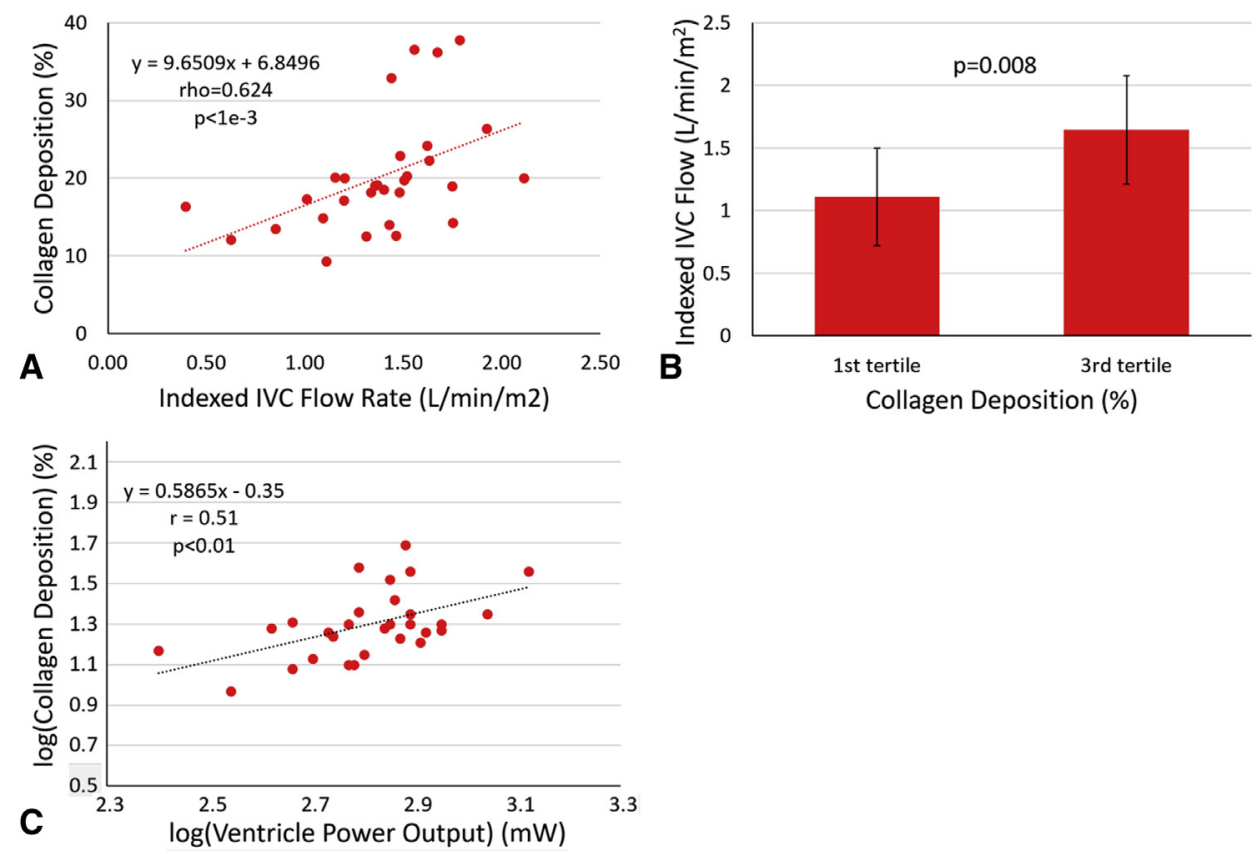

FIGURE 1. Effect of fibrosis on IVC flow and ventricle power output: A, Correlation between IVC flow and percent collagen deposition. B, Collagen deposition tertile comparison. C, Correlation between percent collagen deposition and ventricle power output. IVC, Inferior vena cava. 
TABLE 2. Correlations between hemodynamics and percent collagen deposition

\begin{tabular}{|c|c|c|c|}
\hline & Mean \pm SD [range] & Correlation coefficient (rho) & $P$ value \\
\hline Cardiac index $\left(\mathrm{L} / \mathrm{min} / \mathrm{m}^{2}\right)$ & $3.2 \pm 0.77[1.6-5.4]$ & 0.307 & .088 \\
\hline $\mathrm{CO}(\mathrm{L} / \mathrm{min})^{*}$ & $4.7 \pm 1.3[2.5-7.5]$ & 0.297 & .062 \\
\hline IVC flow $\left(\mathrm{L} / \mathrm{min} / \mathrm{m}^{2}\right) \dagger$ & $1.4 \pm 0.42[0.39-2.6]$ & 0.624 & $<1 \mathrm{e}-3$ \\
\hline $\mathrm{SVC}$ flow $\left(\mathrm{L} / \mathrm{min} / \mathrm{m}^{2}\right)$ & $0.80 \pm 0.36[0.22-1.8]$ & 0.160 & .390 \\
\hline LPA flow $\left(\mathrm{L} / \mathrm{min} / \mathrm{m}^{2}\right)$ & $0.91 \pm 0.27[0.32-1.7]$ & -0.105 & .575 \\
\hline RPA flow $\left(\mathrm{L} / \mathrm{min} / \mathrm{m}^{2}\right)$ & $1.3 \pm 0.39[0.71-2.4]$ & 0.178 & .329 \\
\hline LPV flow $\left(\mathrm{L} / \mathrm{min} / \mathrm{m}^{2}\right)$ & $1.3 \pm 0.32[0.82-2.3]$ & 0.037 & .849 \\
\hline RPV flow $\left(\mathrm{L} / \mathrm{min} / \mathrm{m}^{2}\right)$ & $1.6 \pm 0.40[1.0-3.1]$ & 0.382 & .041 \\
\hline Collateral flow $\left(\mathrm{L} / \mathrm{min} / \mathrm{m}^{2}\right)$ & $1.07 \pm 0.71[0.0-3.0]$ & 0.091 & .625 \\
\hline IVC pressure (mm Hg) & $12.3 \pm 2.5[6-16]$ & -0.038 & .840 \\
\hline SVC pressure (mm Hg) & $12.2 \pm 2.4[6-16]$ & -0.051 & .787 \\
\hline LPA pressure $(\mathrm{mm} \mathrm{Hg})$ & $11.8 \pm 2.6[6-17]$ & -0.154 & .417 \\
\hline RPA pressure (mm Hg) & $11.8 \pm 2.4[6-16]$ & -0.096 & .607 \\
\hline $\operatorname{SVR}\left(\mathrm{WU} / \mathrm{m}^{2}\right)$ & $20.7 \pm 8.3[10.0-48.4]$ & -0.136 & .472 \\
\hline PVR (WU/m²) & $1.4 \pm 0.6[0.37-2.9]$ & -0.247 & .181 \\
\hline IVC pulsatility index $(\%)$ & $54 \pm 35[19-162]$ & -0.159 & .401 \\
\hline
\end{tabular}

Values are mean \pm standard deviation [range]. $S D$, Standard deviation; $C O$, cardiac output; $I V C$, inferior vena cava; $S V C$, superior vena cava; $L P A$, left pulmonary artery; $R P A$, right pulmonary artery; $L P V$, left pulmonary vein; $R P V$, right pulmonary vein; $S V R$, systemic vascular resistance; $W U$, Wood units; $P V R$, pulmonary vascular resistance. *Signifies a log transformation of the data. $\nmid$ Signifies statistical significance.

\section{Fibrosis and Total Cavopulmonary Connection}

Left pulmonary artery (LPA) diameter was negatively correlated with percent collagen deposition and approached statistical significance (rho $=-0.328, P=.067$ ) (Table 4). There were no significant relationships between percent collagen deposition and IVC, superior vena cava, or RPA size, or the extent of caval or branch pulmonary artery stenosis. Flow efficiency through the TCPC showed no significant correlations with percent collagen deposition (Figure 2). TCPC resistance averaged $0.41 \pm 0.35$ (0.101.63) Wood units. The percentage of total ventricular power output lost in the TCPC was $1.8 \% \pm 1.9 \%(0.24-8.17)$. Indexed TCPC PL averaged $0.11 \pm 0.09$ (0.03-0.48). Particle residence time did not correlate with percent collagen deposition with an average of $0.68 \pm 0.20(0.18-1.11)$ seconds, corresponding to $0.84 \pm 0.26(0.18-1.25)$ cardiac cycles. Velocity field and pressure drop maps are shown for a representative low and high percent collagen deposition case in
Figure 3. Velocity magnitudes and pressure drops were slightly higher in the more severe fibrosis case; however, Figure 3 shows only a single time point in the cardiac cycle. Overall, no consistent, discernable differences in flow collisions, flow field features, streamlining, or pressure drop between low and high fibrosis cases were observed.

\section{DISCUSSION}

Liver fibrosis is a serious complication of the current treatment strategy for Fontan survivors. In these patients, although achieving the benefits of reduced intracardiac mixing and increased oxygen saturation, this physiology comes at the obligatory cost of creating a state of chronically elevated CVP. Given that venous congestion likely plays a primary role in this hepatopathy, we sought to investigate whether additional higher-order variables such as flow hemodynamics, efficiency, or pathway architecture might influence the degree of fibrosis seen by using CMR-derived

TABLE 3. Correlations between ventricular function and percent collagen deposition

\begin{tabular}{lccc}
\hline & Mean \pm SD [range] & Correlation coefficient (rho) & $P$ value \\
\hline EDV $\left(\mathrm{mL} / \mathrm{m}^{2}\right)$ & $106 \pm 33[45-181]$ & 0.0061 & .974 \\
End-systolic volume $\left(\mathrm{mL} / \mathrm{m}^{2}\right)$ & $50 \pm 27[7-125]$ & 0.015 & .940 \\
Stroke volume $\left(\mathrm{mL} / \mathrm{m}^{2}\right)$ & $56 \pm 14[35-87]$ & 0.143 & .460 \\
Ejection fraction $(\%)$ & $55 \pm 12[27-85]$ & 0.038 & .846 \\
Ventricular power output $(\mathrm{mW}) *, \dagger$ & $676 \pm 227[253-1317]$ & 0.51 & $<.01$ \\
\hline
\end{tabular}

Values are mean \pm standard deviation [range]. $S D$, Standard deviation; $E D V$, end-diastolic volume. *Signifies statistical significance. †Signifies a log transformation of the data. A Pearson correlation was used for the transformed data. 
TABLE 4. Correlations among total cavopulmonary connection geometry, energetics, and percent collagen deposition

\begin{tabular}{lccc}
\hline & Mean \pm SD [range] & Correlation coefficient (rho) & $P$ value \\
\hline IVC diameter $\left(\mathrm{mm} / \mathrm{m}^{2}\right)$ & $11.6 \pm 2.9[6.4-21.4]$ & -0.058 & .753 \\
SVC diameter $\left(\mathrm{mm} / \mathrm{m}^{2}\right)$ & $9.4 \pm 2.5[5.9-17.0]$ & -0.142 & .438 \\
\hline LPA diameter $\left(\mathrm{mm} / \mathrm{m}^{2}\right)$ & $7.1 \pm 1.1[4.8-9.6]$ & -0.328 & .067 \\
RPA diameter $\left(\mathrm{mm} / \mathrm{m}^{2}\right)$ & $7.4 \pm 2.1[3.9-14.2]$ & 0.016 & .933 \\
IVC stenosis $(\%)$ & $33 \pm 13[10-58]$ & 0.012 & .946 \\
SVC stenosis $(\%)$ & $29 \pm 15[3-56]$ & -0.180 & .324 \\
LPA stenosis $(\%)$ & $51 \pm 18[22-85]$ & 0.112 & .543 \\
RPA stenosis $(\%)$ & $33 \pm 14[1-66]$ & -0.025 & .892 \\
IVC area $\left(\mathrm{cm} / \mathrm{m}^{2}\right)$ & $1.58 \pm 0.61[0.56-2.72]$ & -0.025 & .892 \\
TCPC resistance $(\mathrm{WU})$ & $0.41 \pm 0.35[0.10-1.62]$ & -0.077 & .687 \\
TCPC PL $(\%$ of total power output) & $1.8 \pm 1.9[0.24-8.2]$ & 0.079 & .688 \\
TCPC iPL & $0.11 \pm 0.09[0.03-4.9]$ & 0.062 & .746 \\
Particle residence time $(\mathrm{s})$ & $0.68 \pm 0.20[0.18-1.12]$ & 0.186 & .307 \\
\hline V
\end{tabular}

Values are mean \pm standard deviation [range]. $S D$, Standard deviation; $I V C$, inferior vena cava; $S V C$, superior vena cava; $L P A$, left pulmonary artery; $R P A$, right pulmonary artery; $T C P C$, total cavopulmonary connection; $W U$, Wood units; $P L$, power loss; $i P L$, indexed power loss.

ventricular function, flows, and computational flow dynamics.

Our study is particularly valuable because we have the unique opportunity of analyzing contemporaneous liver biopsy data in conjunction with detailed blood flow characteristics in each patient. The use of an accurate, highresolution liver fibrosis quantification technique is necessary when investigating potential causes and risk factors of liver fibrosis. In this study, liver biopsy samples were analyzed in a unique manner specific to Fontan disease by quantifying percent Sirius Red collagen staining. ${ }^{6,10}$ This method of quantification allows for a more incremental, quantitative scale, which may be more sensitive to teasing out possible associative or causative processes than traditional, graded scoring systems developed for other (non-Fontan) fibrosis mechanisms.
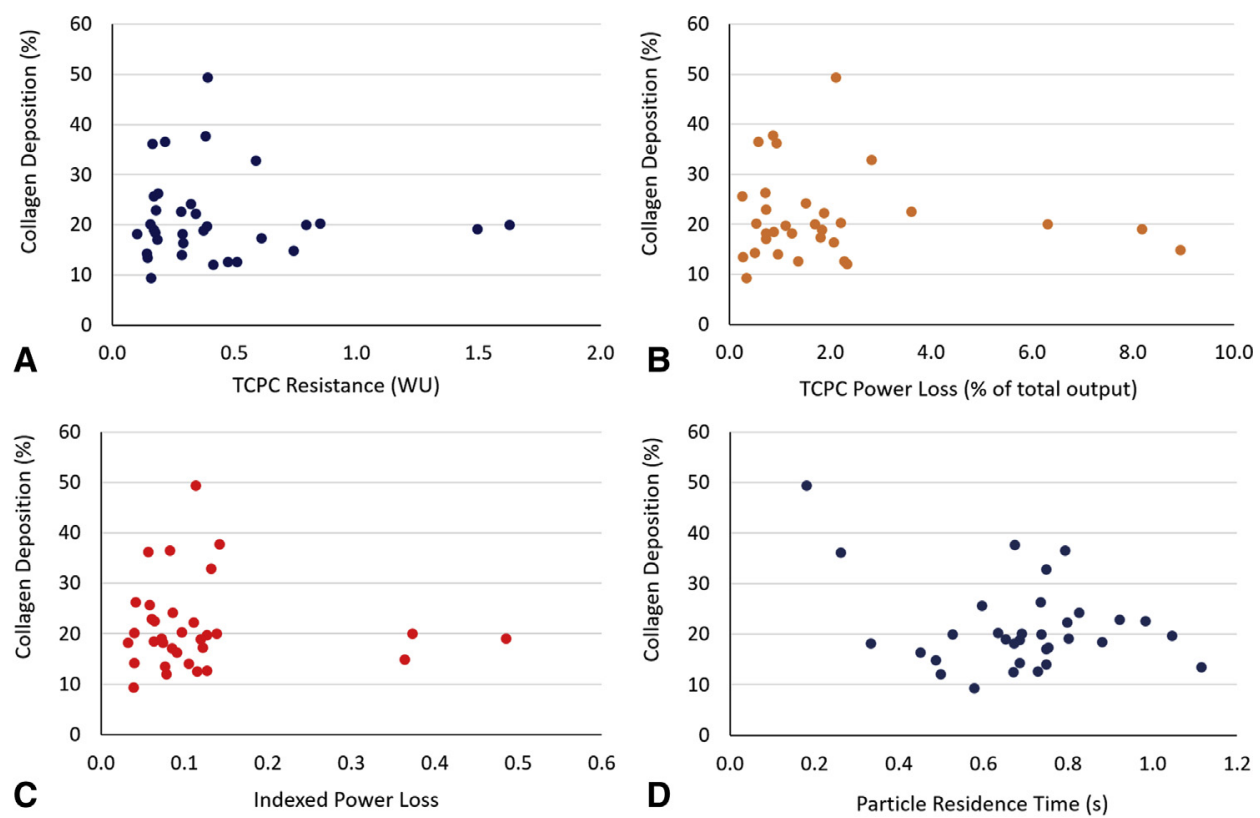

FIGURE 2. Relationship between TCPC energetics and liver fibrosis: Flow efficiency through the TCPC showed no significant correlations with percent collagen deposition. A, TCPC resistance. B, TCPC PL (as percent of total power output). C, Indexed PL. D, Particle residence time. TCPC, Total cavopulmonary connection; $W U$, wood units. 


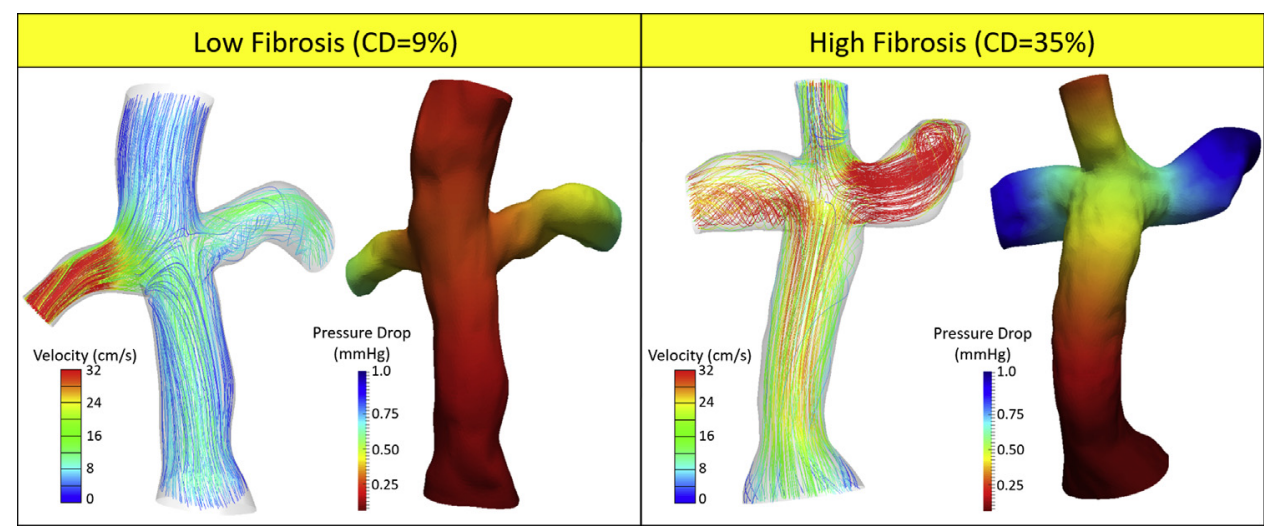

FIGURE 3. Total cavopulmonary connection velocity and pressure fields. Velocity field and pressure drop maps are shown for a representative low and high percent collagen deposition case. Overall, no consistent, discernable differences in flow collisions, flow field features, streamlining, or pressure drop between low and high fibrosis cases were observed. $C D$, Collagen deposition.

This study demonstrated that in patients with single ventricles exposed to Fontan physiology for a mean of approximately 14 years, significant positive associations exist between IVC flow (measured suprahepatic) and ventricular power output. In addition, there was a significant positive correlation with $\mathrm{CO}$. No significant associations were demonstrated with regard to ventricular volumes, caval or branch pulmonary artery or pulmonary venous flow, or TCPC efficiency parameters.

Our study findings do not support the notion that finer aspects of flow hemodynamics through the Fontan circulation mitigate the presence of liver fibrosis. From the architectural perspective, LPA diameter was modestly inversely associated with liver fibrosis. Of note, no patient had clinically important TCPC pathway obstruction present as ascertained through cardiac catheterization and angiography. LPA narrowing is a common finding in many cases of hypoplastic left heart syndrome as the vessel courses behind the somewhat large reconstructed aorta and may theoretically contribute to overall TCPC flow impedance. ${ }^{18}$ However, we found that Fontan energetics were not associated with

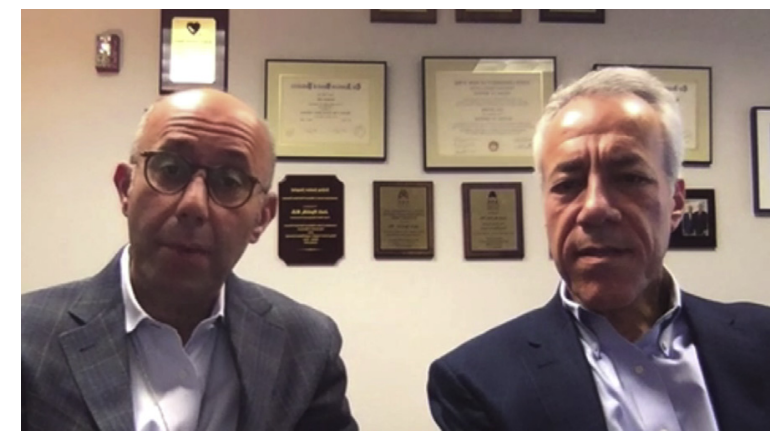

VIDEO 1. Liver fibrosis in patients with single ventricles after the Fontan is related to increased IVC flow and ventricular power output rather than local TCPC hemodynamics. Video available at: http://www.jtcvsonline. org/article/S0022-5223(18)30619-6/fulltext. hepatic fibrosis, suggesting that Fontan pathway architecture and TCPC inefficiency are not the key factors contributing to the degree of fibrosis. In this cohort, the average percentage of total ventricle power output lost in the TCPC was $1.8 \% \pm 1.9 \%$, with a maximum of $8.2 \%$. Therefore, incremental differences in flow kinetics (resistance, PL, particle residence time) due to individual vessel flow rates and surgical TCPC design result in only minor differences that cannot overcome the overriding physiologic issue of elevated venous pressure leading to hepatic congestion. Of note, the similar degrees of hepatic fibrosis in both the most efficient and most inefficient TCPCs suggest that further efforts to optimize Fontan pathway architecture and TCPC design may not be accompanied by delayed progression of liver disease for future Fontan cases.

A positive association was seen between the degree of hepatic fibrosis and the indexed IVC flow rate, as well as $\mathrm{CO}$. We speculate that this is due to hepatic arterialization and thus increased hepatic venous return contributing to IVC flow and CO. Hypervascular nodules and hepatic arterialization have been described in the patient with Fontan circulation, with localization of hypervascularity to the peripheral perfusion "watershed" areas of the liver. ${ }^{8}$ This may reflect adaptation and attempt toward increased blood flow to regions of hypoperfusion or relative hypoxia, perhaps somewhat similar to the phenomenon of aortopulmonary collateral flow seen. ${ }^{19}$ Liver angiogenesis and fibrogenesis have been linked, with a complex interplay between the 2 processes based on the primary mechanism causing the fibrosis. ${ }^{20}$ Although the precise mechanism is unclear, hepatic fibrosis after the Fontan operation may lead to increased hepatic blood flow and increased venous return, and thus may be a source for excessive volume loading of the single ventricle, again similar to systemic to pulmonary collateral flow or valve insufficiency scenarios. This notion requires further investigation as discussed in Video 1. This idea dovetails with the finding of 
increased ventricular power output with increasing hepatic fibrosis. Progressive hepatic fibrosis may be a source for future ventricular failure.

\section{Study Limitations}

All results in this study are based on concurrent CMR, catheterization, and liver biopsy data sets. However, detailed magnetic resonance imaging assessment of the liver was not undertaken, and thus findings such as hypervascular nodularity could not be determined. As we demonstrate in the current study, IVC flow rate is associated with hepatic fibrosis. Therefore, hemodynamic metrics that are dependent on IVC flow rates may change as a result of fibrosis. Consequently, if prior flow rates or TCPC energetics (highly dependent on IVC flow rate) are a factor in future fibrosis progression (eg, Fontan hemodynamics are predictive of future hepatic fibrosis), the use of concurrent data sets would not elucidate this relationship. To address this possibility, a current effort is under way to analyze CMR-derived flow dynamics and CFD energetics at a time point before the liver biopsy. All CFD simulations used rigid walls. Although vessel walls such as the branch pulmonary arteries are generally compliant, this is a common practice and is expected to have only minimal effects on bulk hemodynamic metrics. ${ }^{21}$ In addition, because the TCPC is created from noncompliant materials (eg, polytetrafluoroethylene [Gore-Tex, WL Gore \& Associates, Flagstaff, Ariz]) and 2 to 3 staged surgeries would indeed cause fibrosis around systemic venous pathway vessels, rigid modeling is an acceptable assumption. Some variability is expected with liver biopsy data based on heterogeneity of the disease and sampling bias; however, the current method remains the best available. ${ }^{22}$ Finally, all blood flow characteristics of the TCPC circuit were obtained with the patient at rest, in a supine position. It is plausible to suspect that Fontan hemodynamics are different with the patient upright, ambulatory, and in particular during exercise. Perhaps our finding of LPA size in association with liver fibrosis may suggest that important TCPC flow disturbances might be discovered in association with fibrosis if study could be undertaken in a more natural active daily state or under provocative testing.

\section{CONCLUSIONS}

Liver fibrosis long term after the Fontan operation is positively associated with increased IVC flow, CO, and ventricular power output, which may be due to hepatic arterialization and increased hepatic venous return. In addition, the one anatomic variable that was associated with hepatic fibrosis was LPA diameter. By using a quantitative measure of collagen deposition, liver fibrosis is not associated with CFD-derived parameters such as efficiency, TCPC resistance, or energy loss. Our findings suggest that as they relate to development of liver fibrosis, subtleties in TCPC-related flow dynamics may not be sufficient to overcome the fundamental process of venous congestion and the "congestive hepatopathy" inherently present in every patient with a Fontan circulation. Other factors besides TCPC flow-related characteristics may influence the variability in degree of fibrosis seen after the Fontan operation. In addition to its potentially deleterious impact on the hepatic functions of synthesis and metabolic clearance, progressive liver fibrosis also may be a cause for increased volume load to the single ventricle heart, which may raise additional challenges for our patients as they enter their adult years.

\section{Conflict of Interest Statement}

M.F. reports a research grant, modest from Siemens and Edwards Lifesciences. All other authors have nothing to disclose with regard to commercial support.

\section{References}

1. Gewillig M, Brown SC. The Fontan circulation after 45 years: update in physiology. Heart. 2016;102:1081-6.

2. Pundi KN, Johnson JN, Dearani JA, Pundi KN, Li Z, Hinck ZA, et al. 40-year follow-up after the Fontan operation long-term outcomes of 1,052 patients. $J$ Am Coll Cardiol. 2015;66:1700-10.

3. Rychik J, Goldberg D, Rand E, Semeao E, Russo P, Dori Y, et al. End-organ consequences of the Fontan operation: liver fibrosis, protein-losing enteropathy and plastic bronchitis. Cardiol Young. 2013;23:831-40.

4. Gewillig MH, Lundström UR, Deanfield JE, Bull C, Franklin RC, Graham TP, et al. Impact of Fontan operation on left ventricular size and contractility in tricuspid atresia. Circulation. 1990;81:118-27.

5. Rychik J, Veldtman G, Rand E, Russo P, Rome JJ, Krok K, et al. The precarious state of the liver after a Fontan operation: summary of a multidisciplinary symposium. Pediatr Cardiol. 2012;33:1001-12.

6. Goldberg DJ, Surrey LF, Glatz AC, Dodds K, O'Byrne ML, Lin HC, et al. Hepatic fibrosis is universal following Fontan operation, and severity is associated with time from surgery: a liver biopsy and hemodynamic study. J Am Heart Assoc. 2017;6:e004809.

7. Mertens L, Hagler DJ, Sauer U, Somerville J, Gewillig M. Protein-losing enteropathy after the Fontan operation: an international multicenter study. PLE study group. J Thorac Cardiovasc Surg. 1998;115:1063-73.

8. Kiesewetter CH, Sheron N, Vettukattill JJ, Hacking N, Stedman B, MillwardSadler H, et al. Hepatic changes in the failing Fontan circulation. Heart. 2007; 93:579-84.

9. Lindsay I, Johnson J, Everitt MD, Hoffman J, Yetman AT. Impact of liver disease after the Fontan operation. Am J Cardiol. 2015;115:249-52.

10. Surrey LF, Russo P, Rychik J, Goldberg DJ, Dodds K, O’Byrne ML, et al. Prevalence and characterization of fibrosis in surveillance liver biopsies of patients with Fontan circulation. Hum Pathol. 2016;57:106-15.

11. Narkewicz MR, Sondheimer HM, Ziegler JW, Otanni Y, Lorts A, Shaffer EM, et al. Hepatic dysfunction following the Fontan procedure. J Pediatr Gastroenterol Nutr. 2003;36:352-7.

12. Procelewska M, Kolcz J, Januszewska K, Mroczek T, Malec E. Coagulation abnormalities and liver function after hemi-Fontan and Fontan procedures - the importance of hemodynamics in the early postoperative period. Eur J Cardiothorac Surg. 2007;31:866-72.

13. de Moraes TF, Amorim PHJ, Azevedo FS, da Silva JVL. InVesalius-an opensource imaging application. Comput Vis Med Image Process. 2011;405.

14. Martins T, Barbara A, Silva G, Faria T, Dalava B, Silva J. InVesalius: threedimensional medical reconstruction software. Virtual Rapid Manuf. 2007;135-41.

15. Heiberg E, Sjögren J, Ugander M, Carlsson M, Engblom H, Arheden H. Design and validation of Segment - freely available software for cardiovascular image analysis. BMC Med Imaging. 2010;10:1.

16. Bidhult SL, Carlsson M, Steding-Ehrenborg K, Arheden H, Heiberg E. A new method for vessel segmentation based on a priori input from medical expertise in cine phase-contrast magnetic resonance imaging. J Cardiovasc Magn Reson. 2014;16(Suppl 1):P355.

17. Dasi LP, Pekkan K, Katajima HD, Yoganathan AP. Functional analysis of Fontan energy dissipation. J Biomech. 2008;41:2246-52. 
18. Dasi LP, Sundareswaran KS, Sherwin C, de Zilicourt D, Kanter K, Fogel MA, et al. Larger aortic reconstruction corresponds to diminished left pulmonary artery size in patients with single-ventricle physiology. J Thorac Cardiovasc Surg. 2010;139:557-61.

19. Whitehead KK, Harris MA, Glatz AC, Gillespie MJ, DiMaria MV, Harrison NE, et al. Status of systemic to pulmonary arterial collateral flow after the Fontan procedure. Am J Cardiol. 2015;115:1739-45.

20. Bocca C, Novo E, Miglietta A, Parola M. Angiogenesis and fibrogenesis in chronic liver diseases. Cell Mol Gastroenterol Hepatol. 2015;1:477-88.
21. Patricia L, Susan L. Computational fluid-structure interaction: methods and application to a total cavopulmonary connection. Comput Mech. 2007; 46:69.

22. Germani G, Hytiroglou P, Fotiadu A, Burroughs AK, Dhillon AP. Assessment of fibrosis and cirrhosis in liver biopsies. Semin Liver Dis. 2015:1-3.

Key Words: congenital heart defect, fibrosis, Fontan, hemodynamics, single ventricle 